\title{
Utility of a stent pusher catheter as a "mini-over- tube" in the endoscopic management of post-liver transplant biliary strictures
}
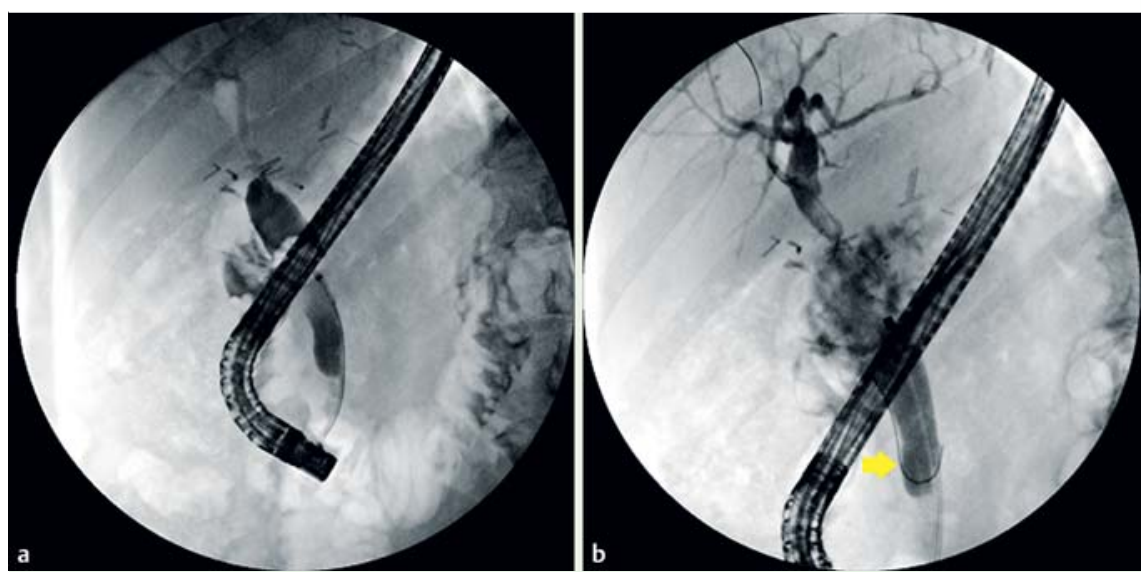

Fig. 1 a Cholangiography showing a severe and complex anastomotic stricture following a liver transplant. $\mathbf{b}$ Only one guidewire could be passed through the stricture: the second wire has flipped back (yellow arrow).
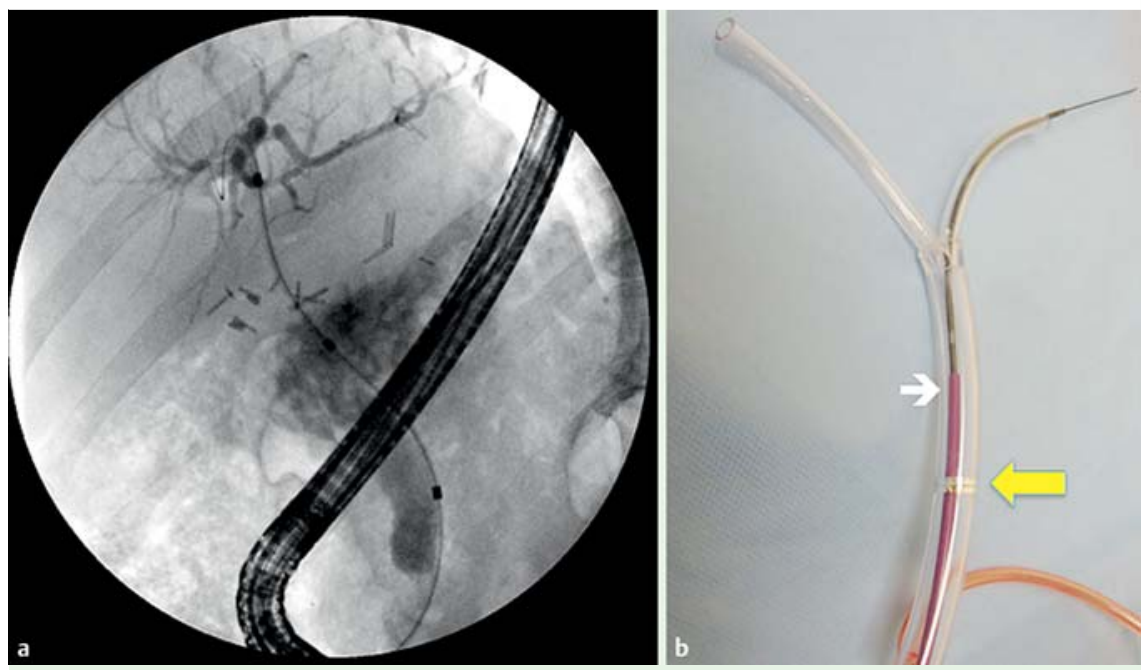

Fig. 2 a Insertion of a pusher tube over the first guidewire. The pusher tube would serve as a "miniovertube." b Illustration of the technique. Once the pusher tube (white arrow) was above the stricture (yellow arrow), the inner guiding catheter would be removed. A second wire could then be advanced through the mini-overtube (i.e., the pusher tube).

Biliary strictures and biliary leaks are the most common forms of post-transplant biliary complication [1-3]. These are thought to be due to multiple factors including ischemia, ischemia-reperfusion injuries, cytomegalovirus infection, and surgical techniques [2]. Endoscopic retrograde cholangiopancreatography (ERCP), percutaneous transhepatic cholangiography, and surgery are the primary approaches used in addressing strictures, with ERCP being the gold standard as a diagnostic and therapeutic tool with the use of balloon dilations and placement of multiple parallel plastic stents [1-3]. However, some strictures have proven to be technically challenging or untreatable, mandating the use of percutaneous transhepatic cholangiographic drainage or even surgical hepaticojejunostomy. Herein we report a new method to deliver two wires above an impassable and severe anastomotic stricture by utilizing the pusher catheter as a "mini-overtube."

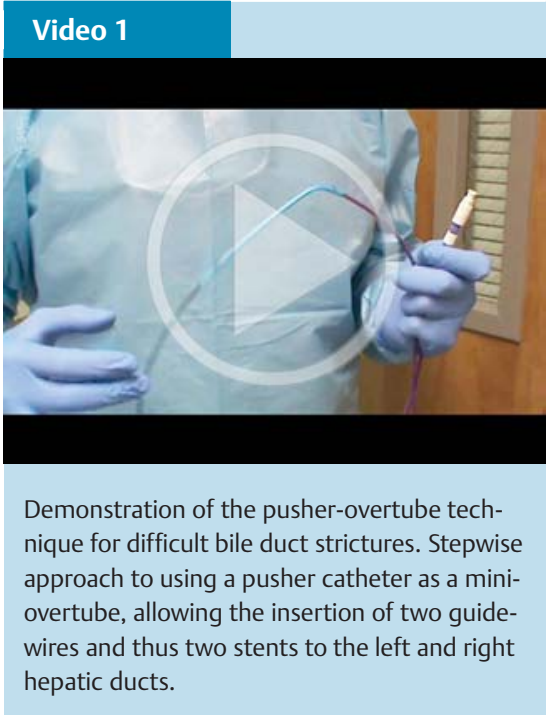

A 55-year-old man had undergone orthotopic liver transplantation (OLT) because of hepatitis $C$ virus-related cirrhosis and hepatocellular carcinoma. After the OLT he had elevated results in liver function tests but biopsy had shown a viable liver transplant. Therefore the patient had undergone ERCP, which had shown an anastomotic biliary stricture. This had been managed by sphincterotomy and placement of a single $10-\mathrm{Fr} 12-\mathrm{cm}$ plastic stent because of the severity of the stricture.

At follow-up ERCP, the previous stent was removed using a snare. Biliary sphincterotomy and balloon dilation of the stenosis was performed (6-mm Titan balloon; Cook Medical, Winston Salem, North Carolina, USA) with removal of debris and sludge and extraction of the balloon without complications. However, on further evaluation only a single 0.035-inch guidewire (Metro; Cook Medical) could be advanced through the anastomosis ( Fig. 1). Several techniques and accessories were used without success in the attempt to pass a second wire: a tapered tip catheter, various sphincterotomes, balloons, and straight and J-tipped wires.

Because the path was so narrow and tortuous, an alternative approach was pursued as follows. A stent delivery device (Cook Medical), namely the inner guiding catheter and pusher without the stent, was passed over the existing 

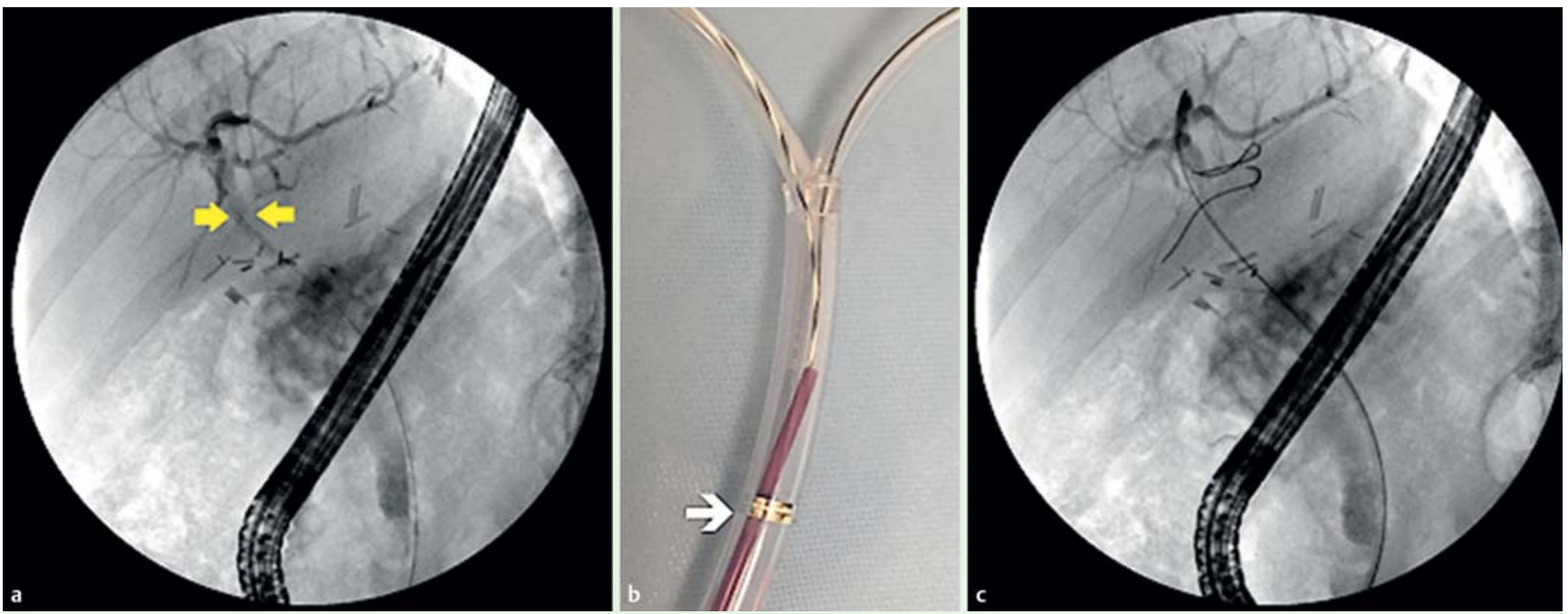

Fig. 3 a Cholangiography showing the pusher tube above the stricture. The yellow arrows show the upper end of the pusher tube. $\mathbf{b}$ Illustration of the technique. The "above-the-stricture" (white arrow) location of the pusher tube allows the the placement of two or more wires. c Cholangiography shows two wires above the stenosis.
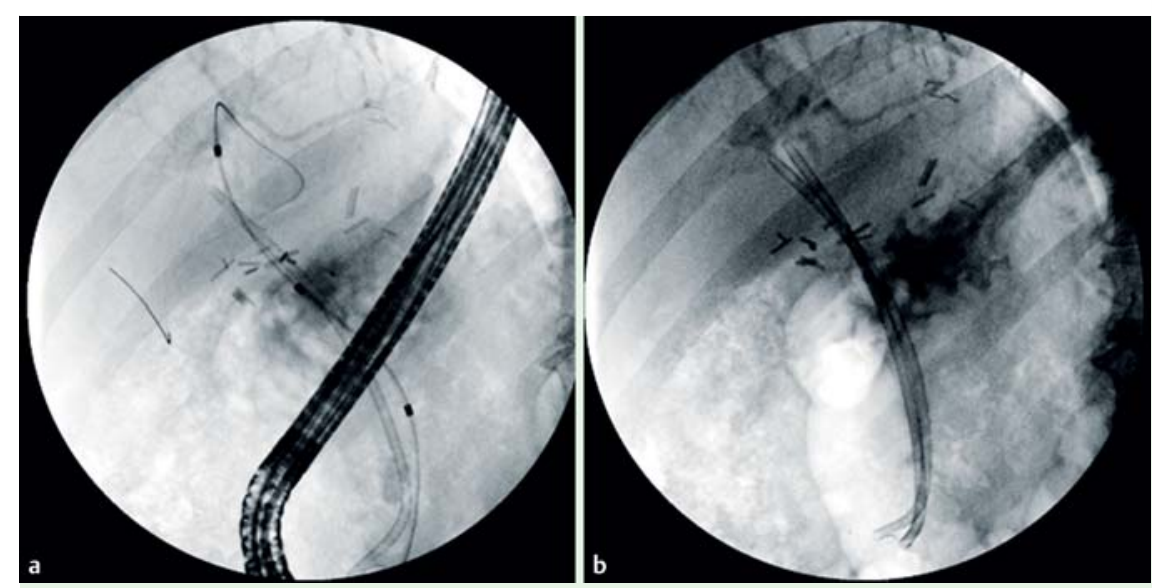

Fig. 4 a Deployment of two 10-Fr plastic stents. b Final appearance of the deployed stents.

guidewire that had crossed the stricture proximally ( $\bullet$ Fig. 2 a, $\bullet$ Video 1$)$. Once the tip of the pusher was above the stricture ( $\bullet$ Fig. $\mathbf{2 b}$ ), the inner guiding catheter was removed, leaving the pusher in situ ( $\bullet$ Video $\mathbf{1})$. The pusher could thus be utilized as a "mini-overtube" allowing the advancement of a second wire inside across the severe anastomotic stricture, so that now two wires could be delivered above the stenosis, one each into the right and left hepatic ducts ( $\odot$ Fig. $\mathbf{3 b}, \odot$ Video 1 ). The pusher tube was then withdrawn, thus allowing the successful insertion of two $10-\mathrm{Fr} 12-\mathrm{cm}$ plastic stents (Cotton-Huibregtse; Cook Medical), one along each wire with both stents crossing the stricture ( $\bullet$ Fig.3, ○ Video 1).
On subsequent ERCP, dilation of the stenosis was more easily obtained (8-mm balloon, Titan; Cook Medical), and again successful insertion of two plastic stents, one along each wire with both stents crossing the stricture (10-Fr Solus plastic double-pigtail 4-cm; $12-\mathrm{cm}$ Cotton-Huibregste Amsterdam plastic stent) (० Fig.4, 0 Video 1 ).

In summary, we have presented a novel method that allows passage of two (or more) wires above a tight biliary stricture, thus permitting the use of more than one stent to treat a complex stricture. The concept of "mini-overtube" in ERCP is useful as it expands the therapeutic possibilities in complex cases such as the one presented.
Endoscopy_UCTN_Code_TTT_1AR_2AG

Competing interests: None

\section{G. Bruno Pereira, Juan P. Gutierrez, Marco D'Assuncao, Lucia C. Fry, Klaus Mönkemüller}

Basil I. Hirschowitz Endoscopic Center of Excellence, Division of Gastroenterology and Hepatology, University of Alabama at Birmingham, Alabama, USA

\section{Acknowledgments \\ $\nabla$}

An American Society for Gastrointestinal Endoscopy (ASGE) research grant supported this work. Klaus Mönkemüller, MD, PhD, FASGE, is the 2014 recipient of an ASGE Research Award for the development of ERCP teaching models. Marco D'Assuncao, MD, MSc, TSOBED, is a Visiting Professor at the University of Alabama at Birmingham, USA. This work was done during Prof. D'Assuncao's professorship at the Basil I. Hirschowitz Endoscopic Center of Excellence, University of Alabama, Birmingham, Alabama, USA. 


\section{References}

1 Ryu CH, Lee SK. Biliary strictures after liver transplantation. Gut Liver 2011; 5: $133-$ 142

2 Seehofer $D$, Eurich $D$, Veltzke-Schlieker $W$ et al. Biliary complications after liver transplantation: old problems and new challenges. Am J Transplant 2013; 13: 253-265

3 Ayoub WS, Esquivel CO, Martin P. Biliary complications following liver transplantation. Dig Dis Sci 2010; 55: 1540-1546
Bibliography

DOI http://dx.doi.org/

10.1055/s-0034-1393386

Endoscopy 2015; 47: E587-E589

(c) Georg Thieme Verlag KG

Stuttgart · New York

ISSN 0013-726X

\section{Corresponding author}

Klaus Mönkemüller, MD, PhD

Division of Gastroenterology and Hepatology Basil I. Hirschowitz Endoscopic

Center of Excellence

Endoscopy Unit, JT 664

619 19th Street $S$

Birmingham, AL 35249

USA

Fax: +1-205-934-1537

klaus1@uab.edu 